\title{
Network Pharmacology Study and Molecular Docking on the Mechanism of Treatment of Colorectal Cancer by Xiao-Chai-Hu-Tang
}

Jingyun Jin

Shanghai University of Traditional Chinese Medicine

Bin Chen

Shanghai PuTuo District Center Hospital

Xiangyang Zhan

Shanghai University of Traditional Chinese Medicine

Zhiyi Zhou

Shanghai University of Traditional Chinese Medicine

Hui Liu

Shanghai University of Traditional Chinese Medicine

Yun Dong ( $\nabla$ dongyun0429@126.com )

Shanghai Chest Hospital: Shanghai Jiao Tong University Affiliated Chest Hospital

https://orcid.org/0000-0003-4286-2701

Research

Keywords: Colorectal cancer, Network pharmacology, Molecular docking, Traditional Chinese Medicine

Posted Date: February 4th, 2021

DOl: https://doi.org/10.21203/rs.3.rs-157805/v1

License: (c) (i) This work is licensed under a Creative Commons Attribution 4.0 International License.

Read Full License 


\section{Abstract}

Background and objective: To predict the targets and signal pathways of Xiao-Chai-Hu-Tang (XCHT) in the treatment of colorectal cancer (CRC) based on network pharmacology, to further analyze its anti-CRC material basis and mechanism of action.

Methods: TCMSP and TCMID databases were adopted to screen the active ingredients and potential targets of XCHT. CRC-related targets were retrieved by analyzing published microarray data (accession number GSE110224) from the Gene Expression Omnibus (GEO) database. The above common targets were used to construct the "herb-active ingredients-target" network by Cytoscape 3.8.0 software. And

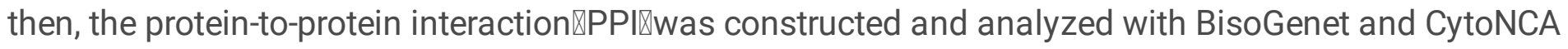
plug-in in Cytoscape. Gene Ontology (GO) functional and the Kyoto Encyclopaedia of Genes and Genomes (KEGG) pathway enrichment analysis for target genes were then performed using the $R$ package of cluster Profiler. Further, AutoDock Vina software was used to conduct molecular docking studies on the active ingredients and key targets to verify the network pharmacological analysis results.

Results: A total of 71 active ingredients of XCHT and 20 potential targets for anti-CRC were identified. The network analysis revealed that quercetin, stigmasterol, kaempferol, baicalein, acacetin may be the key compounds. And PTGS2, NR3C2, CA2, MMP1 may be the key targets. The active ingredients of XCHT interacted with most disease targets of CRC. It fully showed that XCHT exerted its therapeutic effect through the synergistic action of the multi-compound, multi-target, and multi-pathway. Gene ontology enrichment analysis showed $46 \mathrm{GO}$ entries, including 20 biological processes, 6 cellular components, and 20 molecular functions. 11 KEGG signaling pathways had been identified, including IL-17 signaling pathway, TNF signaling pathway, Toll-like receptor signaling pathway, and NF-kappa B signaling pathway. It showed that $\mathrm{XCHT}$ played a role in the treatment of $\mathrm{CRC}$ by regulating different signal pathways. Molecular docking confirmed the correlation between five core compounds (including quercetin, stigmasterol, kaempferol, baicalein, and acacetin) and PTGS2.

Conclusion: The potential active ingredients, possible targets, and key biological pathways for the efficacy of XCHT in the treatment of CRC were preliminarily described, which provided a theoretical basis for further experimental verification research.

\section{Introduction}

Colorectal cancer (CRC) is the third most common malignancy in the world and the fourth leading cause of cancer death in the world ${ }^{[1]}$. In recent years, the incidence and mortality rates of colorectal cancer have increased significantly in developing countries ${ }^{[2]}$. At present, the treatment of CRC is mainly based on surgery, radiotherapy, and chemotherapy with TCM. TCM has been widely accepted worldwide as an essential complementary medicine that provides beneficial therapeutic effects for cancer patients ${ }^{[3]}$. In America, Chinese herbal medicine has been used as an auxiliary medicine for cancer treatment ${ }^{[4]}$. Previous studies have shown that traditional herbal medicine as adjuvant therapy, combined with 
chemotherapy or radiation therapy, can improve the therapeutic effect, reduce adverse effects, improve quality of life, and prolong survival time ${ }^{[5-9]}$.

Xiao-Chai-Hu-Tang (XCHT) is derived from the Treatise on Febrile and Miscellaneous Diseases and is an extract of seven herbs: Radix Bupleuri (Chai-Hu), Pinellia ternata (Ban-Xia), Scutellaria baicalensis (Huang-Qin or Chinese skullcap root), Zizyphus jujube (Da-Zao or jujube fruit), Panax ginseng (Ren-Shen or ginseng root), Zingiber officinale (Sheng-Jiang or ginger rhizome) and Prepared Liauorice Root (ZhiGan-Cao). Studies have shown that XCHT can treat tumors by enhancing immune regulation, antiangiogenesis, and tumor cell apoptosis ${ }^{[10,11]}$. XCHT and its plus or minus formula have an excellent experimental and clinical basis in malignant tumors such as gastric cancer ${ }^{[12]}$, lung cancer ${ }^{[13]}$, breast cancer $^{[14]}$, liver cancer ${ }^{[15]}$, and it also has a good prospect in CRC. Some studies have shown ${ }^{[16]}$ that XCHT may inhibit CRC progression in mice with chronic stress CRC model by inhibiting NLRP3 inflammasome activity and pro-inflammatory factor secretion and down-regulating NF-kB, but the specifically related mechanism is still unclear.

Network pharmacology is a new strategy to identify the mechanism of herb-ingredient-target multimolecular synergy at the systemic level based on the network viewpoint ${ }^{[17]}$. Therefore, this study predicts the target and signaling pathways of $\mathrm{XCHT}$ against $\mathrm{CRC}$ from the perspective of network pharmacology and further analyzes its anti-CRC material basis and mechanism of action. Besides, molecular docking technology was used for verification, to provide a theoretical foundation for further study and reasonable clinical application of XCHT against CRC.(Fig. 1)

\section{Materials And Methods}

\subsection{Chemical Compounds and Targets for XCHT.}

With the keywords of "ChaiHu", "HuangQin", "BanXia", "DaZao", "ShengJiang", "RenShen" and "ZhiGanCao", the active ingredients of $\mathrm{XCHT}$ and its corresponding targets were obtained in the Traditional Chinese Medicine Systems Pharmacology Database(TCMSP, http://Isp.nwu.edu.cn/, Ver.2.3)and the Traditional Chinese Medicine Integrated Database (TCMID, http://www.megabionet.org/tcmid/, Ver.1.0). In this study, oral bioavailability (OB) and drug-likeness $(\mathrm{DL})$ were adopted to identify the bioactive ingredients of $\mathrm{XCHT}$. Only compounds that had related targets and met the ADME criteria (ie, OB threshold $>30 \%$ and DL thresh-old $>0.18$ ) were included in the study for subsequent research. Then, restricting the species to humans, Uniprot KB function of Uniprot (http://www.uniprot.org/)database was used to retrieve and match targets of compounds. Compounds without corresponding targets and redundant duplicates were removed. Eventually, the targets of active components were obtained and converted into gene symbols.

\subsection{Identification Differentially Expressed Genes of CRC}

The microarray expression profile dataset GSE110224 was downloaded from the National Center for Biotechnology Information (NCBI) GEO (http://www.ncbi.nlm.nih.gov/geo/) database, which is based on 
the GPL570 [HG-U133_Plus_2] Affymetrix Human GenomeU133 Plus 2.0 Array platform. The dataset contained 34 samples, including 17 primary colorectal cancer tissue samples and 17 matched normal tissue samples. The script was run in Strawberry Perl-5.30.2.1 (Perl) software, and the gene probe names were annotated as gene symbols and grouped. Then, R packages ("limma" and "pheatmap") were used to analyze GSE110224. Differentially expressed genes(DEGs)between normal tissue and colorectal cancer tissue were detected on the basis of the criteria adjusted P-value $<0.05$ and $|\log F C|>1$, and were visualized using a volcano plot.

\subsection{Herb-Active Ingredient-Target Interaction Network Construction}

The action targets of XCHT and the DEGs of CRC were taken as intersection, and the intersection targets of these two parts are defined as the key targets of XCHT in the treatment of CRC. The herb-active ingredient-target interaction network was visualized using Cytoscape 3.8.0 software (www.cytoscape.org/). Taking disease $\square$ key targets $\square$ herbs $\square$ active ingredients as nodes, the corresponding relationship above all were constructed with Excel and then imported into the software of Cytoscape 3.8.0 to construct the interaction network of $\mathrm{XCHT}$ in the treatment of $\mathrm{CRC}$. Then, the Analyze Network plug-in in Cytoscape was used to calculate and sort the topological parameters (degree) of the network.

\subsection{Construction and Analysis of the Protein-Protein Interaction Network}

The PPI network was constructed using the BisoGenet plug-in in Cytoscape. And CytoNCA, a Cytoscape plugin for network centrality analysis, was used to identify crucial genes in the network. Firstly, Genes with the highest degree centrality (DC) values in the top 30\% were selected for subnetwork construction using CytoNCA. Then, genes with the top $30 \%$ highest value of betweenness centrality (BC) in the subnetwork were identified as key genes and formed the core network.

\subsection{GO and KEGG Pathway Enrichment.}

Go biological function analysis is mainly used to describe the functions of gene targets, including molecular function (MF), cellular component (CC), biological process (BP); KEGG enrichment analysis can obtain the signaling pathways enriched by common targets of XCHT and CRC. GO and KEGG Pathways Enrichment were constructed using the R packages ("clusterProfiler," "org.Hs.eg.db," "enrichplot," and "ggplot2") based on the criteria p-value cutoff $=0.05$, q-value cutoff $=0.05$ ).

\subsection{Molecular Docking}

Molecular docking was performed by using Autodock Vina ${ }^{[18,19]}$. The atomic coordinates of crucial target were retrieved from the Protein Data Bank (www.rcsb.org) and were prepared in AutoDockTools-1.5.6 by removing water molecules, adding charge, and parameterizing. The 3D structures of active ingredients were downloaded from Traditional Chinese Medicine Systems Pharmacology Database and Analysis Platform (www.tcmspnw.com), and prepared in AutoDockTools by computing atomic partial charges and 
parameterizing. The docking site was set in a cubic box in the center of initial ligand, and a grid map of each atom type in the box was computed. All compounds and the residues within $5 \AA$ of initial ligand were set as flexible while other residues on protein were considered as a rigid template. The initial ligand was also retrieved and redock into the protein with the same protocol. The best scoring conformer of each compound was analyzed and visualized in PYMOL ${ }^{[20,21]}$.

\section{Results}

\subsection{Active ingredients and potential targets of XCHT}

In this study, the TCMSP database was used to search for all the compounds of XCHT, and 124 active compounds were selected based on $\mathrm{OB}>30 \%$ and $\mathrm{DL}>0.18$. Corresponding relationships between the active ingredients and the targets, deleting duplicate targets, and converting targets into Gene Symbol using Uniprot, 82 active compounds and 238 targets were obtained after removing the chemical compounds that lack potential targets information. There were 12 active compounds for Radix Bupleuri, 32 for Scutellaria baicalensis, 2 for Prepared Liauorice Root, 17 for Panax ginseng, 11 for Pinellia ternata, 4 for Zingiber Officinale, and 18 for Zizyphus jujube. (Supplementary file, Table S1)

\subsection{Screening of DEGs in CRC}

By analyzing the GSE110224 dataset derived from the GEO database, 519 DEGs were obtained basing on the cutoff criteria, including 296 downregulated and 223 upregulated genes. The volcano map and heatmap of the distribution of DEGs are shown in Fig. 2,3. The top 10 significantly upregulated and downregulated DEGs are listed in Table 1.

Table.1 The identified top 10 upregulated and downregulated DEGs between CRC tissue samples and normal tissue samples 


\begin{tabular}{|llllll|}
\hline $\begin{array}{l}\text { Gene } \\
\text { name }\end{array}$ & $\begin{array}{l}\text { Upregulated } \\
\text { DEGs }\end{array}$ & $\begin{array}{l}\text { Adjusted P } \\
\text { value }\end{array}$ & $\begin{array}{l}\text { Gene } \\
\text { name }\end{array}$ & $\begin{array}{l}\text { Downregulated } \\
\text { DEGs }\end{array}$ & $\begin{array}{l}\text { Adjusted P } \\
\text { value }\end{array}$ \\
REP3 & LogFC & $1.21 \mathrm{E}-03$ & CLCA4 & LogFC & $4.39 \mathrm{E}-03$ \\
FOXQ1 & 4.1151 & $5.87 \mathrm{E}-03$ & MS4A12 & -4.4301 & $3.91 \mathrm{E}-03$ \\
CXCL11 & 3.8374 & $1.83 \mathrm{E}-03$ & AQP8 & -4.4268 & $1.88 \mathrm{E}-03$ \\
MMP7 & 3.5542 & $1.98 \mathrm{E}-03$ & CA4 & -4.2887 & $2.13 \mathrm{E}-03$ \\
REG1B & 3.3532 & $1.58 \mathrm{E}-03$ & GCG & -3.6018 & $9.92 \mathrm{E}-03$ \\
TCN1 & 3.3007 & $2.89 \mathrm{E}-02$ & GUCA2A & -3.5859 & $3.80 \mathrm{E}-03$ \\
MMP1 & 3.2331 & $3.36 \mathrm{E}-03$ & CLDN8 & -3.3854 & $1.16 \mathrm{E}-02$ \\
SLC6A14 & 3.2274 & $2.77 \mathrm{E}-03$ & CHP2 & -3.3201 & $1.68 \mathrm{E}-03$ \\
CXCL3 & 2.7038 & $6.51 \mathrm{E}-03$ & NXPE4 & -3.1559 & $3.22 \mathrm{E}-03$ \\
& 2.6319 & $5.25 \mathrm{E}-03$ & MAMDC2 & -3.1142 & $3.26 \mathrm{E}-03$ \\
\hline 3
\end{tabular}

\subsection{Construction and analysis of the " Herb-Active ingredient-Target " network of XCHT}

After intersecting the $238 \mathrm{XCHT}$-related targets with 519 DEGs of CRC, 20 genes were acquired as the crucial genes of XCHT against CRC (Supplementary file, Table S2). "Herb-Active ingredient-Target" Network of XCHT against CRC was constructed by Cytoscape software (Fig. 4). There are 98 nodes (71 compound nodes, 20 target gene nodes, and 7 herbs) and 191 edges of the network, of which pink circles stand for the herbs of XCHT, green quadrangles for active ingredients, and the blue diamonds for potential targets. The links between the nodes represent the functional relationships of these nodes. The higher the number of connected nodes, the more critical the role of the targets or compounds becomes in this network. After calculating the degree of the network by the Analyze - Network. We could find that quercetin, stigmasterol, kaempferol, baicalein, and acacetin were the top 5 in terms of the degree of all the active ingredients. Besides, the gene PTGS2 is the gene associated with the highest number of active ingredients, followed by NR3C2, CA2, and MMP1, TNFSF15, CCNB1(Supplementary file, Table S3).

Figure 4 shows that there are interactions between the active ingredients of $\mathrm{XCHT}$ and most of the targets of CRC. It fully illustrates that XCHT exerts a therapeutic effect through the synergistic actions of multiple compounds, multiple targets, and multiple pathways. Among them, quercetin, stigmasterol had more interactions, suggesting that these active ingredients play a crucial role in the mechanism of XCHT against CRC.

\subsection{CRC Targets' PPI Network Analysis}


A PPI network of XCHT-CRC target genes comprised 444 nodes and 3,271 edges (Fig. 5A), and the subnetwork was acquired through selecting the top $30 \%$ degree centrality (DC), which consisted of 134 nodes and 1,619 edges (Fig. 5B). After screening the top 30\% betweenness centrality (BC) of the subnetwork, the core network was constructed, which included 41 nodes and 341 edges (Fig. 5C). According to the core network, two target genes, CCNB1 and SPP1, were defined as the critical genes in the core network of PPI, further verifying the crucial role of the two proteins.

\subsection{GO and Pathway Enrichment Analyses.}

To further investigate the mechanism of XCHT in the treatment of $\mathrm{CRC}$, the crucial target genes were analyzed by GO biological process enrichment and KEGG pathway enrichment analysis in this study. The GO enrichment analysis includes three parts: biological process (BP), cellular component (CC), and molecular function (MF). 20 potential targets of XCHT for CRC were analyzed by R packages. The top 20 terms of BP, MF and 6 terms of CC were shown in Fig. 6-8, Supplementary file, Table S4.

Among the Fig. 6-9, the bubble graphs are on the left and the barplots are on the right. In the barplots below, each bar represents a GO and KEGG term on the vertical axis. The number of genes enriched in each term is recorded on the horizontal axis. Color of each bar represents the adjusted $p$ value of each GO and KEGG term. More red the color of the term is, smaller its adjusted $p$ value is. Similarly, In the bubble graphs below, each bubble represents a GO and KEGG path on the vertical axis. The proportion of the genes is recorded on the horizontal axis. The size of each bubble indicates the number of genes enriched in each GO and KEGG pathway. The larger the bubble, the greater the number of genes involved in the pathway. The color of each bubble represents the adjusted P value for each GO and KEGG path. The redder the bubble, the smaller the adjusted $\mathrm{P}$ value.

The top 11 pathways of KEGG enrichment analysis were shown in Fig. 9, such as IL-17 signaling pathway, TNF signaling pathway, Toll-like receptor signaling pathway, NF-kappa B signaling pathway, etc. The CRC related association information of the pathways was summarized in Supplementary file, Table S5. The targets of the active ingredients of XCHT were distributed in different signaling pathways, coordinated with each other, and exerting their therapeutic effect on CRC by regulating the different signaling pathways. 
Table 2

The docking affinity and interactions of compounds binding to PTGS2

\begin{tabular}{|c|c|c|c|c|c|}
\hline \multirow[t]{2}{*}{ Protein } & \multirow{2}{*}{$\begin{array}{l}\text { PDB } \\
\text { ID }\end{array}$} & \multirow[t]{2}{*}{ Compound } & \multirow{2}{*}{$\begin{array}{l}\text { Affinity } \\
(\mathrm{kcal} / \mathrm{mol})\end{array}$} & \multicolumn{2}{|c|}{ Interacted residues } \\
\hline & & & & $\begin{array}{l}\text { Hydrogen } \\
\text { bond }\end{array}$ & Hydrophobic interaction \\
\hline \multirow[t]{10}{*}{ PTGS2 } & \multirow[t]{10}{*}{$5 \mathrm{IKR}$} & \multirow[t]{2}{*}{ quercetin } & \multirow[t]{2}{*}{-9.3} & Tyr385 & \multirow{2}{*}{$\begin{array}{l}\text { His90, Gln192, Tyr348, Val349, Leu352, } \\
\text { Ser353, Tyr355, Tyr385, Trp387, Ala516, } \\
\text { lle517, Phe518, Val523, Gly526, Ala527, } \\
\text { Ser530 }\end{array}$} \\
\hline & & & & Ser530 & \\
\hline & & \multirow[t]{2}{*}{ kaempferol } & \multirow[t]{2}{*}{-9.4} & Tyr385 & \multirow{2}{*}{$\begin{array}{l}\text { His90, GIn192, Tyr348, Val349, Leu352, } \\
\text { Ser353, Tyr355, Tyr385, Trp387, Ala516, } \\
\text { lle517, Phe518, Val523, Gly526, Ser530 }\end{array}$} \\
\hline & & & & Ser530 & \\
\hline & & stigmasterol & -9.6 & - & $\begin{array}{l}\text { Val89, Leu93, Tyr115, Val116, Ser119, } \\
\text { Arg120, Tyr348, Val349, Leu352, } \\
\text { Ser353, Tyr355, Leu359, Tyr385, Trp387, } \\
\text { Phe518, Val523, Gly526, Ala527, } \\
\text { Ser530, Leu531 }\end{array}$ \\
\hline & & baicalein & -9.3 & Ser530 & $\begin{array}{l}\text { His90, GIn192, Val349, Leu352, Ser353, } \\
\text { Phe381, Leu384, Tyr385, Trp387, } \\
\text { Ala516, Ile517, Phe518, Met522, Val523, } \\
\text { Gly526, Ala527, Ser530 }\end{array}$ \\
\hline & & \multirow[t]{2}{*}{ acacetin } & \multirow[t]{2}{*}{-9.5} & Tyr385 & \multirow{2}{*}{$\begin{array}{l}\text { His90, Gln192, Phe205, Val344, Tyr348, } \\
\text { Val349, Leu352, Ser353, Tyr355, Tyr385, } \\
\text { Trp387, Ala516, Ile517, Phe518, Val523, } \\
\text { Ser530 }\end{array}$} \\
\hline & & & & Ser530 & \\
\hline & & \multirow{2}{*}{$\begin{array}{l}\text { Mefenamic } \\
\text { acid } \\
\text { (Initial } \\
\text { ligand) }\end{array}$} & \multirow[t]{2}{*}{-9.2} & Tyr385 & \multirow{2}{*}{$\begin{array}{l}\text { Val116, Tyr348, Val349, Leu352, Ser353, } \\
\text { Tyr355, Leu359, Phe381, Leu384, } \\
\text { Tyr385, Trp387, Met522, Val523, } \\
\text { Gly526, Ala527, Ser530, Leu531 }\end{array}$} \\
\hline & & & & Ser530 & \\
\hline
\end{tabular}

\subsection{Molecular Docking}

Five active ingredients (including quercetin, stigmasterol, kaempferol, baicalein, and acacetin) and one potential target gene PTGS2, were selected through network analysis, whose scores of node and confidence were high. Molecular docking studies were conducted to identify the interactions between active ingredients of XCHT and CRC-related potential target genes at the molecular level. AutoDock Vina was used for molecular docking. The docking score is shown in Table 2. The docking site was set in a cubic box in the center of initial ligand (box size: $60 \times 60 \times 60 \AA$, spacing: 0.375). The lower the docking score (the greater the negative value), the higher the binding force between the compound and the protein. The molecular docking results showed that the binding force of the original ligand mefenamic acid to PTGS2 was $-9.2 \mathrm{kcal} / \mathrm{mol}$, and the docking scores of quercetin, stigmasterol, kaempferol, baicalein, and acacetin to PTGS2 protein ranged from $-9.3 \mathrm{kcal} / \mathrm{mol}$ to $-9.6 \mathrm{kcal} / \mathrm{mol}$, suggesting that these compounds could have a strong binding effect with PTGS2 protein. 
Further analysis of the compounds binding to PTGS2 protein (Fig. 10) showed that all compounds could bind to the active pockets of PTGS2 and form different degrees of interaction, including hydrophobic interaction with Val349, Leu352, Ser353, Tyr385, Trp387, Val523, Ser530. Quercetin, Kaempferol, Baicalein, and Acacetin could also form hydrogen bonds with Ser530. These interactions are similar to those formed when mefenamic acid binds PTGS2. Although stigmasterol did not form hydrogen bonds with PTGS2, it formed hydrophobic action with more amino acids. These molecular docking results suggested that these compounds could bind stably to the active pocket of PTGS2 protein.

\section{Discussion}

$\mathrm{XCHT}$ is derived from the Treatise on Febrile and Miscellaneous Diseases. Studies have shown that it can treat various malignant tumors, including CRC, but the relevant mechanism is still unclear.

In this study, by analyzing the "herb-active ingredient-target" network, it was found that quercetin, stigmasterol, kaempferol, baicalein, acacetin had a high correlation in the network, and significant anticolorectal cancer effects ${ }^{[22-25]}$. For example, quercetin can inhibit CRC by inhibiting the cell cycle, increasing apoptosis, inhibiting angiogenesis and metastasis ${ }^{[22]}$. Baicalein can inhibit the expression of matrix metalloproteinase-2 (MMP-2) and matrix metalloproteinase-9 (MMP-9) by inhibiting the protein kinase B (Akt) signal pathway, thereby inhibiting the migration and invasion of CRC cells ${ }^{[24]}$. Kaempferol has strong cytotoxicity, anti-oxidant, anti-proliferation, and anti-apoptosis effects on HCT-116, HCT-15, SW480, and other CRC cells ${ }^{[26]}$, and also can be used alone or combined with 5-FU to overcome the drug resistance of $\mathrm{CRC}^{[27]}$. Acacetin induces mitochondrial ROS-mediated cell death in a caspase-independent manner in SW480 and HCT-116 colon carcinoma cells by inducing an apoptosis-inducing factor (AIF) to enhance the therapeutic effect of anti-CRC ${ }^{[28]}$, which may be the material basis of $\mathrm{XCHT}$ in the treatment of CRC.

PTGS2, also known as COX-2, is typically induced by inflammatory stimuli and expressed at high levels in Colorectal Cancer Patients ${ }^{[29]}$. There are observational studies, which indicates that PTGS2 inhibitors could positively affect CRC patients' survival after diagnosis ${ }^{[30-32]}$. NR3C2 is a mineralocorticoid receptor, also known as MR. Research has shown that MR can negatively regulate colorectal tumorigenesis. Besides, expression of MR is causally associated with the decrease of VEGFA expression, and VEGFA supports angiogenesis, which is critical for the growth and progression of solid tumors in a hypoxic environment ${ }^{[33]}$. CA II is highly expressed in different normal organs, but its expression is inhibited in cancer cells ${ }^{[34]}$. Besides, CA II overexpression can improve the CRC cells' sensitivity to chemotherapy drugs, which may be a potential biomarker for early CRC diagnosis ${ }^{[35]}$. Research shows that the expression of MMP-1 in colorectal cancer tissues is higher than that in normal colon tissues, and MMP-1 may influence the occurrence and development of colorectal cancer through EMT and Akt signaling pathways ${ }^{[36]}$. In conclusion, the active ingredients' targets of XCHT interacts with the disease targets of CRC, giving full play to the unique advantages of multi-compounds and multi-targets of traditional Chinese medicine to prevent and treat CRC. 
After PPI network analysis, CCNB1 and SPP1 were defined as the critical genes. CCNB1, also known as cyclin B1, and high expression of CCNB1 may be associated with poor survival in patients with $\mathrm{CRC}^{[37]}$. The expression of SPP1 was up-regulated in CRC cells, and it may be a potential key target for the treatment of $\mathrm{CRC}^{[38]}$.

Through GO enrichment analysis, it is found that the anti-CRC targets of XCHT mainly involve the regulation of neuroinflammatory response, collagen catabolic process, and embryo implantation to play the role of anti-CRC, which reflects the way of traditional Chinese medicine in the treatment of diseases. The results of KEGG enrichment analysis suggest that besides the enrichment in cancer-related pathways, such as prostate cancer, bladder cancer. The targets of the main compounds of XCHT are also concentrated in tumors, signal pathways, and other pathways closely related to CRC, such as Prostate cancer, IL-17 signaling pathway, TNF signaling pathway, Toll-like receptor signaling pathway, NF-kappa B signaling pathway, etc. Relevant studies have shown that IL-17 inhibits CD8 CTLs and Tregs' infiltration by signaling in colorectal tumor cells, thereby promoting CRC development ${ }^{[39]}$. Tumor necrosis factor-a (TNF-a), a cytokine, and an essential inflammatory mediator, plays a vital role in the malignant cellular proliferation, angiogenesis, tissue invasion, and metastasis in $\mathrm{CRC}^{[40]}$. Toll-like receptor (TLR) creates the first line of defense against pathogens and the formation of cancer and plays a crucial role in regulating epithelial proliferative, barrier homeostasis, and apoptotic response ${ }^{[41]}$. NF-kappa B signaling pathway plays a key role in the cell proliferation, apoptosis, angiogenesis, and metastasis of $\mathrm{CRC}^{[42]}$. Besides, the targets of the main compounds of XCHT are also enriched in pathways related to inflammation, including Rheumatoid arthritis, Coronavirus disease COVID-19, etc., suggesting that XCHT may act on a variety of cytokines anti-inflammatory and have an effect on CRC.

According to the " Herb-Active ingredient-Target " network results, we selected 5 active ingredients and one key target gene to docking, and the docking results that these compounds could bind stably to the active pocket of PTGS2 protein, which indicates that XCHT may treat CRC by inhibiting PTGS2.

From the perspective of network pharmacology, this study preliminarily elaborates on the potential active ingredients, possible targets, and critical biological pathways of XCHT in CRC treatment, providing a theoretical basis for further experimental verification. Given the limitations of network pharmacology, the pharmacological mechanism of XCHT in the treatment of $\mathrm{CRC}$ is only predicted by mining data, and the effects of the content of various compounds, the interaction between compounds and the metabolic process of herbs in vivo were ignored, which needs to be further verified through pharmacological and clinical studies.

\section{Conclusion}

In summary, this study demonstrated the potential mechanism of XCHT treated with $\mathrm{CRC}$ based on network pharmacology method and molecular docking technology, namely quercetin, stigmasterol, kaempferol, baicalein, and acacetin played a critical role in colorectal cancer by acting PTGS2. Besides, molecular docking also showed that the main active components of XCHT (quercetin, stigmasterol, 
kaempferol, baicalein, and acacetin) could docked well with PTGS2, which provide an important basis for further exploration. However, this study also has certain limitation, still need to further validated by pharmacological and clinical research.

\section{Declarations}

\section{Acknowledgements}

The authors wish to thank the editor and the anonymous reviewers whose constructive comments are very helpful in strengthening the presentation of this paper. This work was financially supported by the

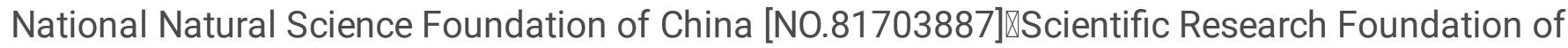
Shanghai Science and Technology Commission [No.18401903000].

\section{Authors' contributions}

JJY, LH: study design; acquisition of data; analysis of data; drafting of the manuscript. CB, DY: acquisition of data; critical revision of the manuscript. ZXY and ZZY: revision of the manuscript and study supervision. All the authors read and approved the final manuscript.

\section{Availability of data and materials}

All data are available in the manuscript and they are exhibited in figures and tables.

\section{Ethics approval and consent to participate}

No applicable. This manuscript does not report on or involve the use of any animal or human data or tissue.

\section{Competing interests}

Authors declare no conflict of interest.

\section{References}

1. Bray F, Ferlay J, Soerjomataram I, et al. Global cancer statistics 2018: GLOBOCAN estimates of incidence and mortality worldwide for 36 cancers in 185 countries. CA Cancer J Clin[J]. 2018;68(6):394-424.

2. Arnold M, Sierra MS, Laversanne M, et al. Global patterns and trends in colorectal cancer incidence and mortality. Gut[J]. 2017;66(4):683-91. 
3. Xiang Y, Guo Z, Zhu P, et al. Traditional Chinese medicine as a cancer treatment: Modern perspectives of ancient but advanced science. Cancer Med[J]. 2019;8(5):1958-75.

4. Wang CZ, Calway T, Yuan CS. Herbal medicines as adjuvants for cancer therapeutics. Am J Chin Med[J]. 2012;40(4):657-69.

5. Mao D, Feng L, Huang S, et al. Meta-Analysis of Xihuang Pill Efficacy When Combined with Chemotherapy for Treatment of Breast Cancer. Evid Based Complement Alternat Med[J]. 2019;2019:3502460.

6. Wang Q, Jiao L, Wang S, et al. Maintenance Chemotherapy With Chinese Herb Medicine Formulas vs. With Placebo in Patients With Advanced Non-small Cell Lung Cancer After First-Line Chemotherapy: A Multicenter, Randomized, Double-Blind Trial. Front Pharmacol[J]. 2018;9:1233.

7. Tang YC, Zhang Y, Zhou J, et al. Ginsenoside Rg3 targets cancer stem cells and tumor angiogenesis to inhibit colorectal cancer progression in vivo. Int J Oncol[J]. 2018;52(1):127-38.

8. Zhang Q, Chen X, Luo Y, et al. Fuzi Enhances Anti-Tumor Efficacy of Radiotherapy on Lung Cancer. J Cancer[J]. 2017;8(19):3945-51.

9. Liu X, Tian S, Liu M, et al. Wogonin inhibits the proliferation and invasion, and induces the apoptosis of HepG2 and Bel7402 HCC cells through NFkappaB/Bcl-2, EGFR and EGFR downstream ERK/AKT signaling. Int J Mol Med[J]. 2016;38(4):1250-6.

10. Han G, Feng J. Application of Xiaochaihu Decoction in preventing and treating cancer. Zhejiang Journal of Traditional Chinese Medicine. 2010;45(09):687-9. (In Chinese).

11. Liao HF, Lu MC, Chang HC, et al. Effects of herbal medicinal formulas on suppressing viral replication and modulating immune responses. Am J Chin Med[J]. 2010;38(1):173-90.

12. Cao W, Pan Y, Zhu Y, et al. Clinical Study of Modified Xiaochaihu Decoction Combined with Emotional Intervention in the Treatment of Gastric Cancer. Journal of Liaoning University of Traditional Chinese Medicine. 2020;22(06):173-6. (In Chinese).

13. ZHAO Y,CHEN M HAOS, et al. Mechanism of Anti- lung Cancer Effect of Xiao Chaihutang Based on Network Pharmacology. Chinese Journal of Experimental Traditional Medical Formulae. 2020;26(09):208-14. (In Chinese).

14. Du J, Jin J, Wu Y. Effect of Xiaochaihu Decoction Combined with Chemotherapy on Immune Function and Quality of Life of Breast Cancer. World Chinese Medicine. 2018;13(06):1452-5 + 1460 . (In Chinese).

15. Zhao J, Liu L, Zhang Y, et al. The Herbal Mixture Xiao-Chai-Hu Tang (Xcht) Induces Apoptosis of Human Hepatocellular Carcinoma Huh7 Cells in Vitro and in Vivo. Afr J Tradit Complement Altern Med[J]. 2017;14(3):231-41.

16. SHAO S, WEN H and ZHANG, Z, et al. Effects of Xiaochaihu Decoction on NF-KB/NLRP3 signaling pathway in mice with stress associated colorectal cancer. Shanghai J Tradit Chin Med. 2020;54(S1):132-6 + 141. (In Chinese).

17. Li S, Zhang B. Traditional Chinese medicine network pharmacology: theory, methodology and application. Chin J Nat Med[J]. 2013;11(2):110-20. 
18. Trott O, Olson AJ. AutoDock Vina: improving the speed and accuracy of docking with a new scoring function, efficient optimization, and multithreading. J Comput Chem. 2010;31:455-61.

19. Kukol, Andreas. Consensus virtual screening approaches to predict protein ligands.[J]. Eur J Med Chem. 2011;46:4661-4.

20. DeLano WL. Use of PYMOL as a communications tool for molecular science., Abstr Pap Am Chem S, 228 (2004) U313-U314.

21. DeLano WL, Lam JW. PyMOL: A communications tool for computational models, Abstr Pap Am Chem S, 230 (2005) U1371-U1372.

22. Darband SG, Kaviani M, Yousefi B, et al. Quercetin: A functional dietary flavonoid with potential chemo-preventive properties in colorectal cancer. J Cell Physiol[J]. 2018;233(9):6544-60.

23. Lu L, Wang Y, Ou R, et al. DACT2 Epigenetic Stimulator Exerts Dual Efficacy for Colorectal Cancer Prevention and Treatment. Pharmacol Res[J]. 2018;129:318-28..- kaempferol.

24. Rui X, Yan XI, Zhang K. Baicalein inhibits the migration and invasion of colorectal cancer cells via suppression of the AKT signaling pathway. Oncol Lett[J]. 2016;11(1):685-8.

25. Li Z, Zhang G, Han C, et al. Anti-cancer Effects and Mechanism of Acacetin. Chinese Archives of Traditional Chinese Medicine.2020:1-8. (In Chinese).

26. Sezer ED, Oktay LM, Karadadas E, et al. Assessing Anticancer Potential of Blueberry Flavonoids, Quercetin, Kaempferol, and Gentisic Acid, Through Oxidative Stress and Apoptosis Parameters on HCT-116 Cells. J Med Food[J]. 2019;22(11):1118-26.

27. Riahi-Chebbi I, Souid S, Othman H, et al. The Phenolic compound Kaempferol overcomes 5fluorouracil resistance in human resistant LS174 colon cancer cells. Sci Rep[J]. 2019;9(1):195.

28. Prasad N, Sharma JR, Yadav UCS. Induction of growth cessation by acacetin via $\beta$-catenin pathway and apoptosis by apoptosis inducing factor activation in colorectal carcinoma cells. Molecular Biology Reports: An International Journal on Molecular and Cellular Biology[J]. 2020;47(3).

29. Rattan NR, Vati RS, Vikas G, et al. Over-Expression of Cyclooxygenase-2 in Colorectal Cancer Patients.[J]. Asian Pac J Cancer Prev. 2019;20:1675-81.

30. Ng Kimmie MJeffreyA, Chan Andrew T, et al. Aspirin and COX-2 inhibitor use in patients with stage III colon cancer.[. J]J Natl Cancer Inst. 2015;107:345.

31. Friis Søren R, Anders H, Erichsen R, et al. Low-Dose Aspirin or Nonsteroidal Anti-inflammatory Drug Use and Colorectal Cancer Risk: A Population-Based, Case-Control Study.[J]. Ann Intern Med. 2015;163:347-55.

32. Xinwei H, Phipps Amanda I, Burnett-Hartman Andrea N, et al. Timing of Aspirin and Other Nonsteroidal Anti-Inflammatory Drug Use Among Patients With Colorectal Cancer in Relation to Tumor Markers and Survival.[. J]J Clin Oncol. 2017;35:2806-13.

33. Tiberio Laura N, Riccardo VV, et al. The decrease of mineralcorticoid receptor drives angiogenic pathways in colorectal cancer.[J]. PLoS One. 2013;8:e59410. 
34. Li X-J, San-Ju XHai-Long,L, et al. Reduction of CAll Expression in Gastric Cancer: Correlation with Invasion and Metastasis.[J]. Chin J Cancer Res. 2012;24:196-200.

35. Zhou R, Huang W, Yao Y, et al. "CA II, a potential biomarker by proteomic analysis, exerts significant inhibitory effect on the growth of colorectal cancer cells" Int J Oncol, vol. 43, no. 2, pp. 611-621.

36. Wang Kai Z, bao J. Yu Junhui et al. Knockdown of MMP-1 inhibits the progression of colorectal cancer by suppressing the PI3K/Akt/c-myc signaling pathway and EMT.[J]. Oncol Rep. 2020;43:1103-12.

37. Liu Xiaoqun L, Xiangdong Q, Tiankui, et al. Identification of crucial genes and pathways associated with colorectal cancer by bioinformatics analysis.[J]. Oncol Lett. 2020;19:1881-9.

38. Fahai GONG, Bao YANG, Shuhao SUN, et al. Study on the Expression Difference of SPP1 in Different CRC Cell Lines and the Regulation Mechanism of EMT[J]. Journal of Ningxia Medical University. 2019;41(06):584-8. (In Chinese).

39. Chen Ju Y, Xiaoyang P, Elise, et al. IL-17 inhibits CXCL9/10-mediated recruitment of CD8 cytotoxic T cells and regulatory $T$ cells to colorectal tumors.[J]. J Immunother Cancer. 2019;7:324.

40. Zafar BM, Mehraj BH, Zeenat $\mathrm{H}$, et al. Tumor necrosis factor-a (TNF-a)-308G/A promoter polymorphism in colorectal cancer in ethnic Kashmiri population - A case control study in a detailed perspective.[J]. Meta Gene. 2016;9:128-36.

41. Sato Yusuke G, Yasufumi N, Norihiko, et al. Cancer Cells Expressing Toll-like Receptors and the Tumor Microenvironment.[J]. Cancer Microenviron, 2009, null: 205 - 14.

42. Patel Meera, Horgan Paul G, McMillan Donald C, et al. NF-kB pathways in the development and progression of colorectal cancer.[J]. Transl Res. 2018;197:43-56.

\section{Figures}




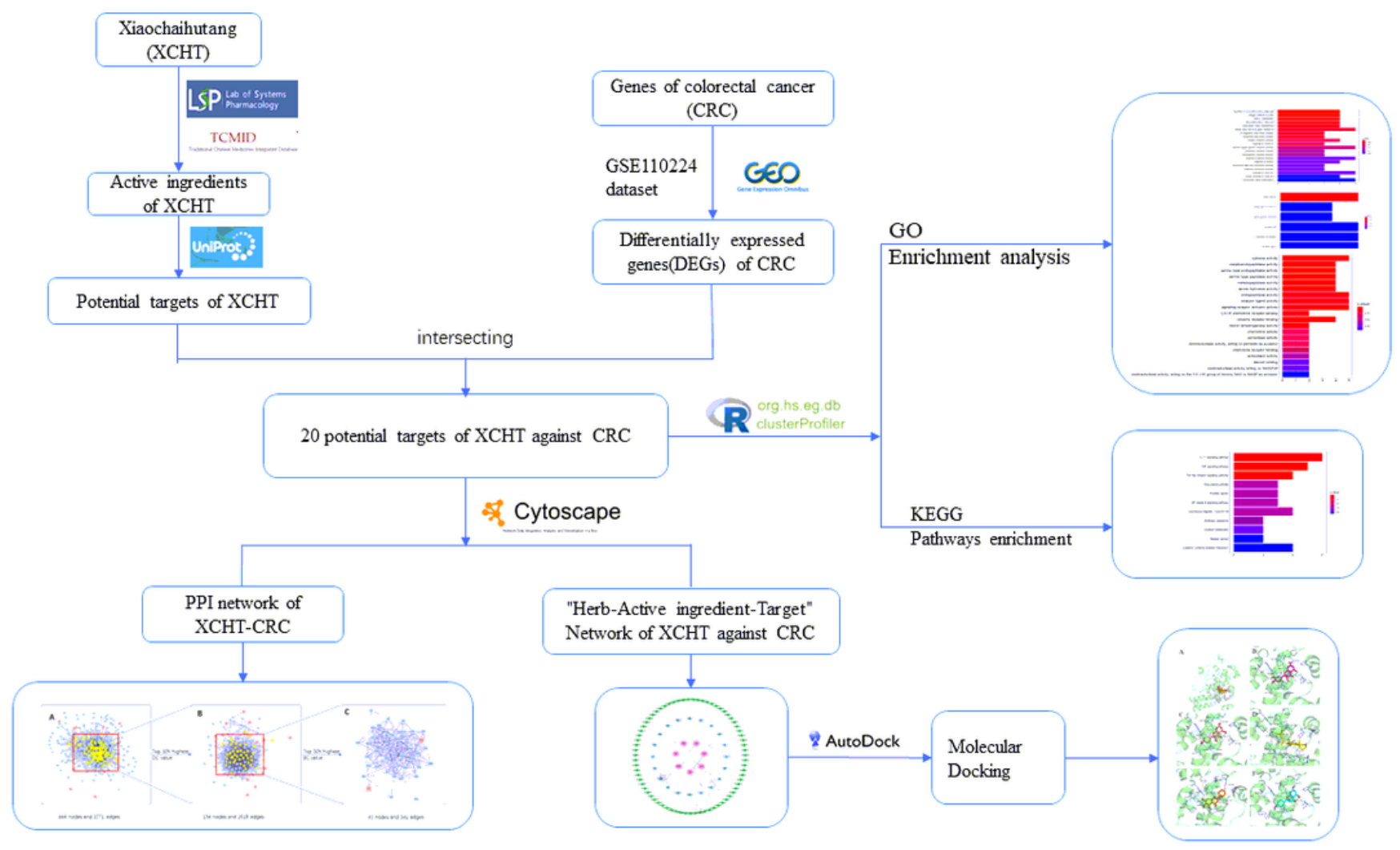

Figure 1

Research process. 


\section{Volcano}

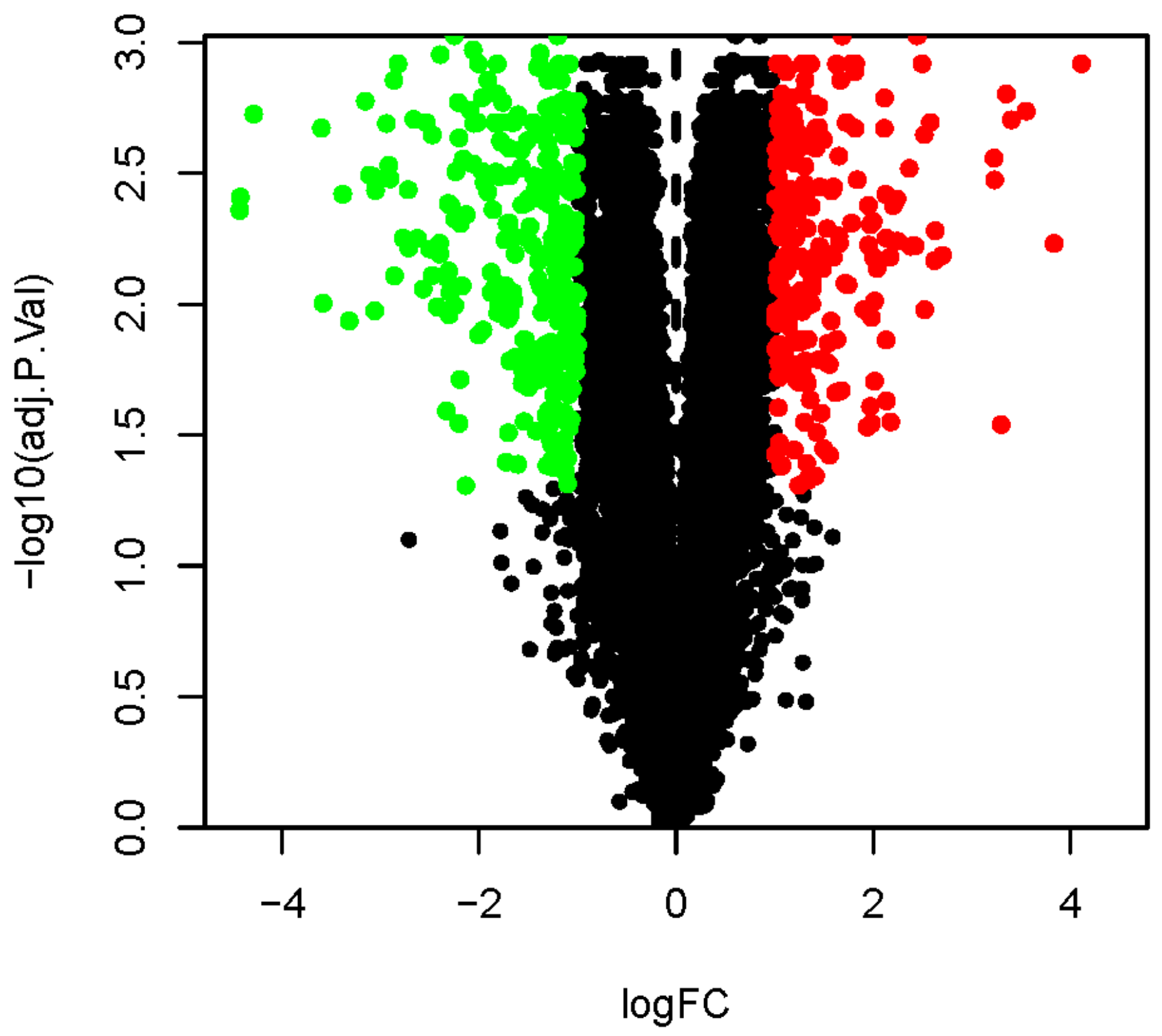

Figure 2

Volcano map of differentially expressed genes. The red and the green dots represent DEGs that were significantly upregulated (genes) and downregulated (genes), respectively (adjusted p-value $<0.05$, and $|\log \mathrm{FC}|>1)$. 


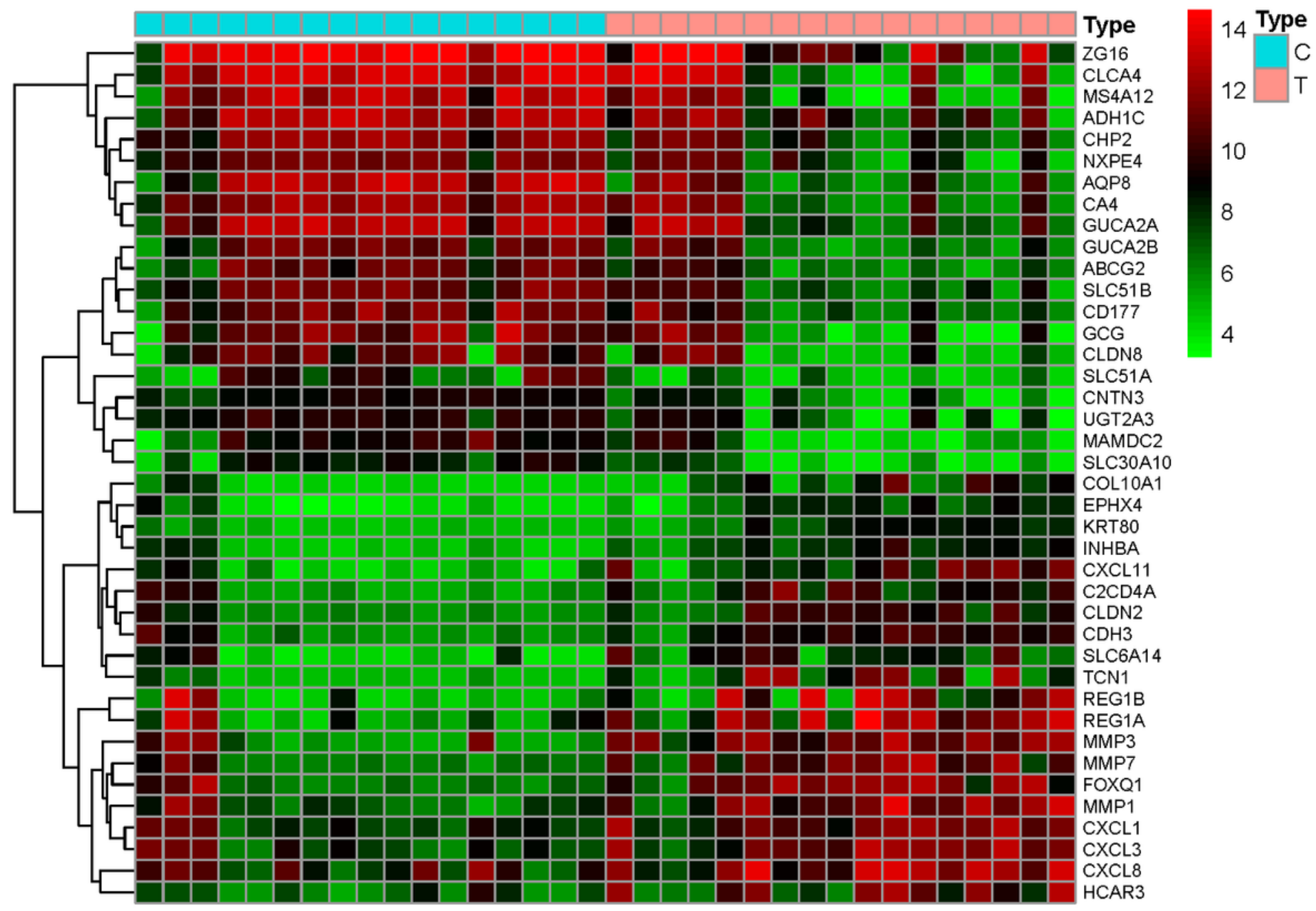

Figure 3

Heatmap of differentially expressed genes. Red indicates that the gene is highly expressed in the sample, and green indicates that the gene has a lower expression in the sample. 


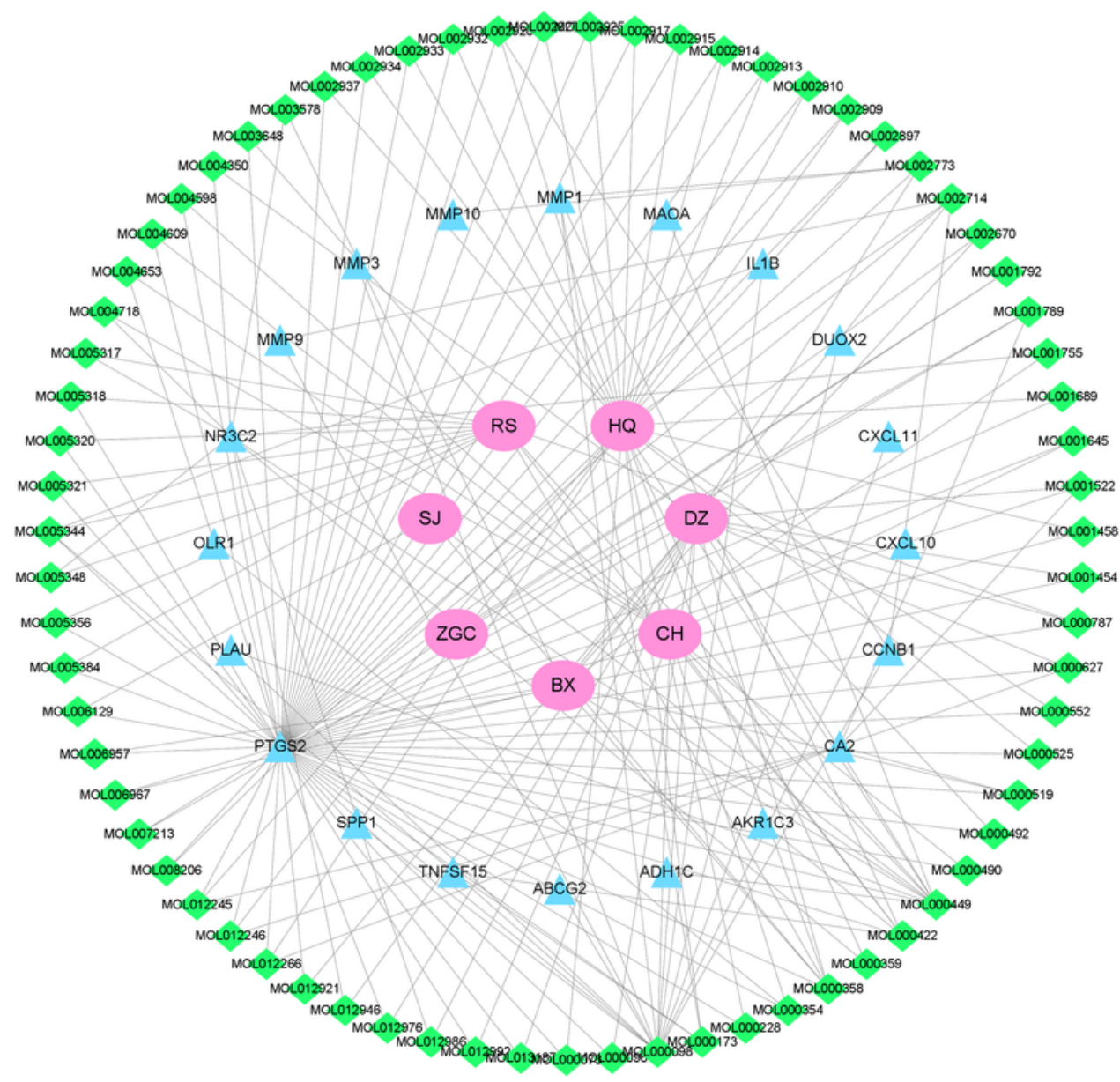

Figure 4

"Herb-active ingredient-target" Network of XCHT against CRC 


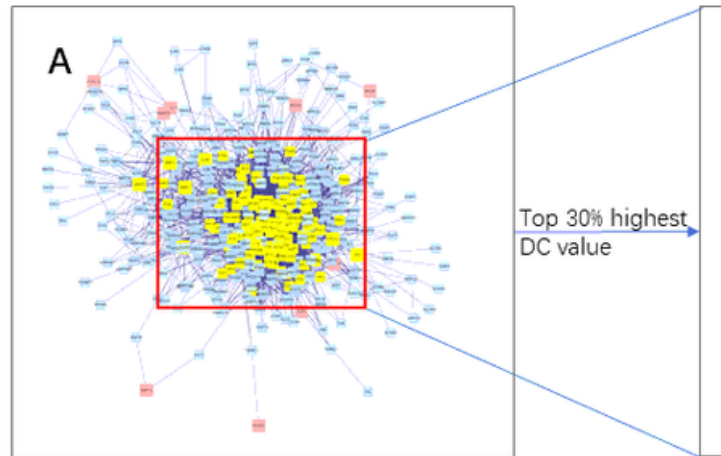

444 nodes and 3271 edges

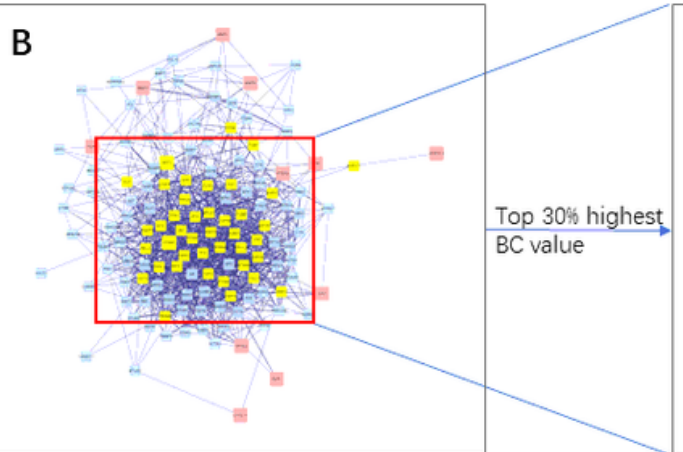

134 nodes and 1619 edges

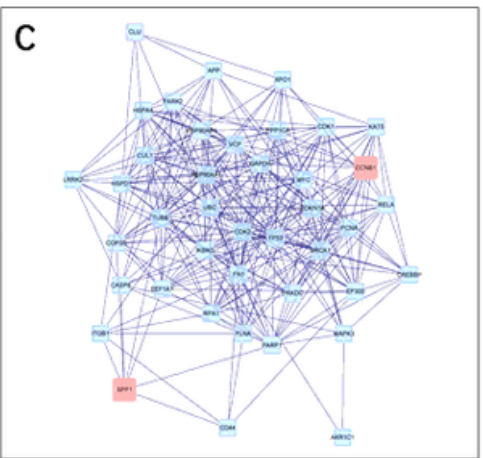

41 nodes and 341 edges

\section{Figure 5}

Topological analysis of the protein-protein interaction network. (A) The PPI network of XCHT-CRC target genes. (B) The PPI network of significant proteins extracted from A. (C) The PPI network of crucial XCHT targets for CRC treatment extracted from B.
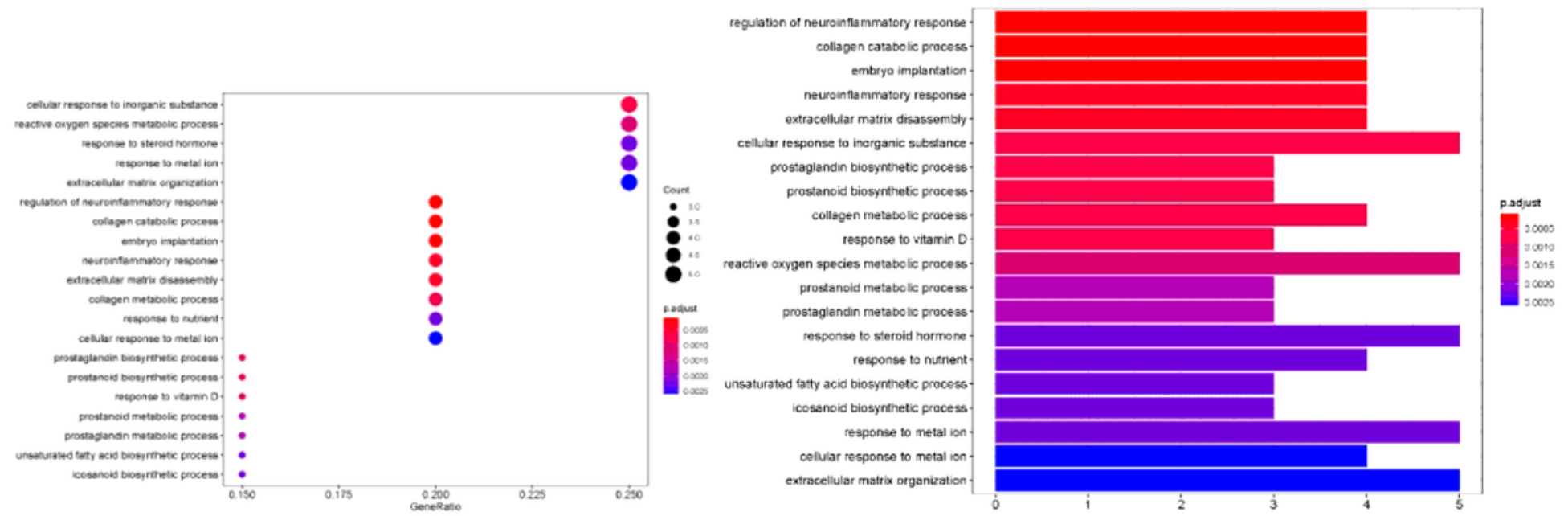

Figure 6

BP analysis of GO enrichment 


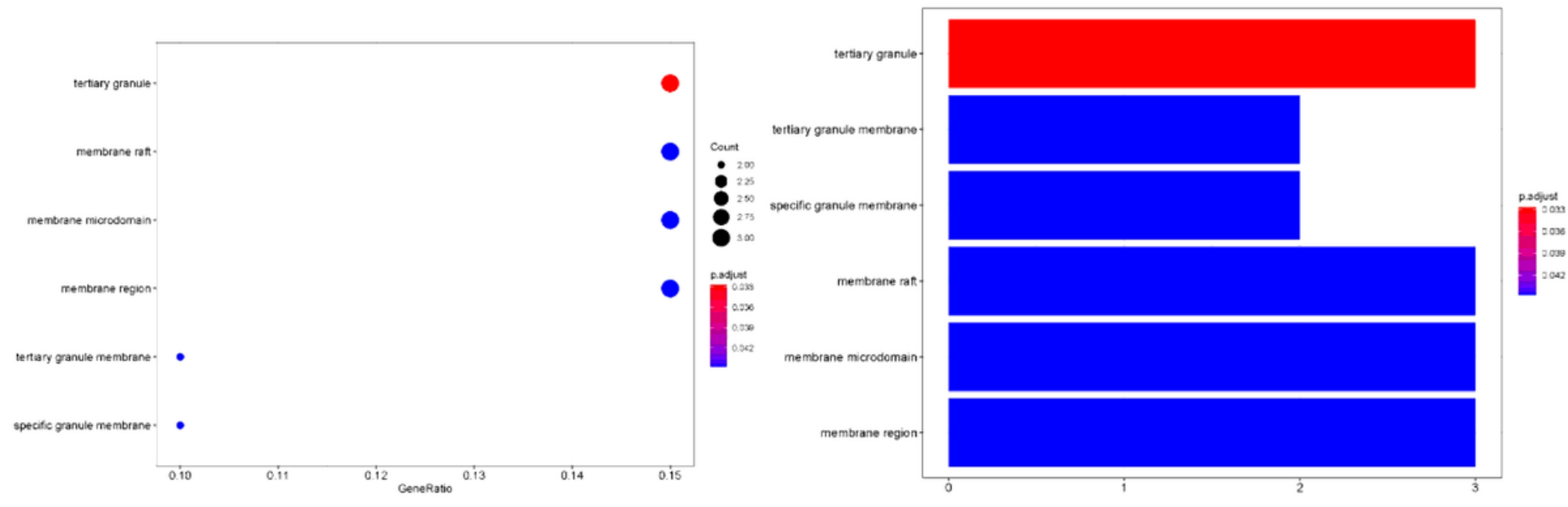

Figure 7

$\mathrm{CC}$ analysis of $\mathrm{GO}$ enrichment
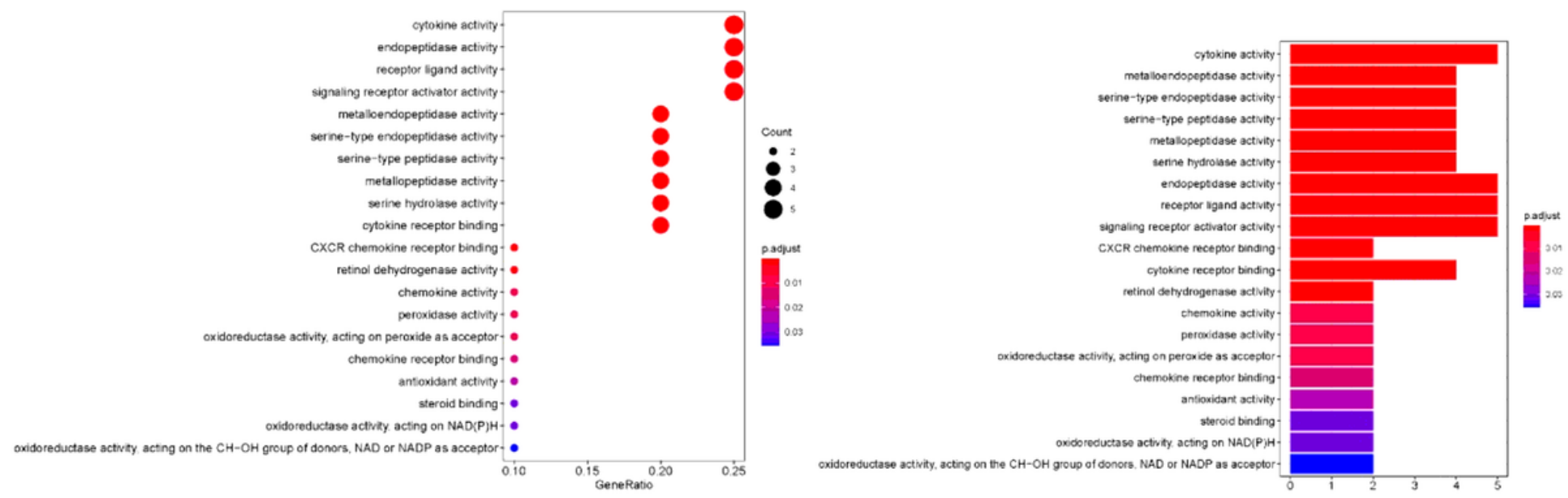

Figure 8

MF analysis of GO enrichment
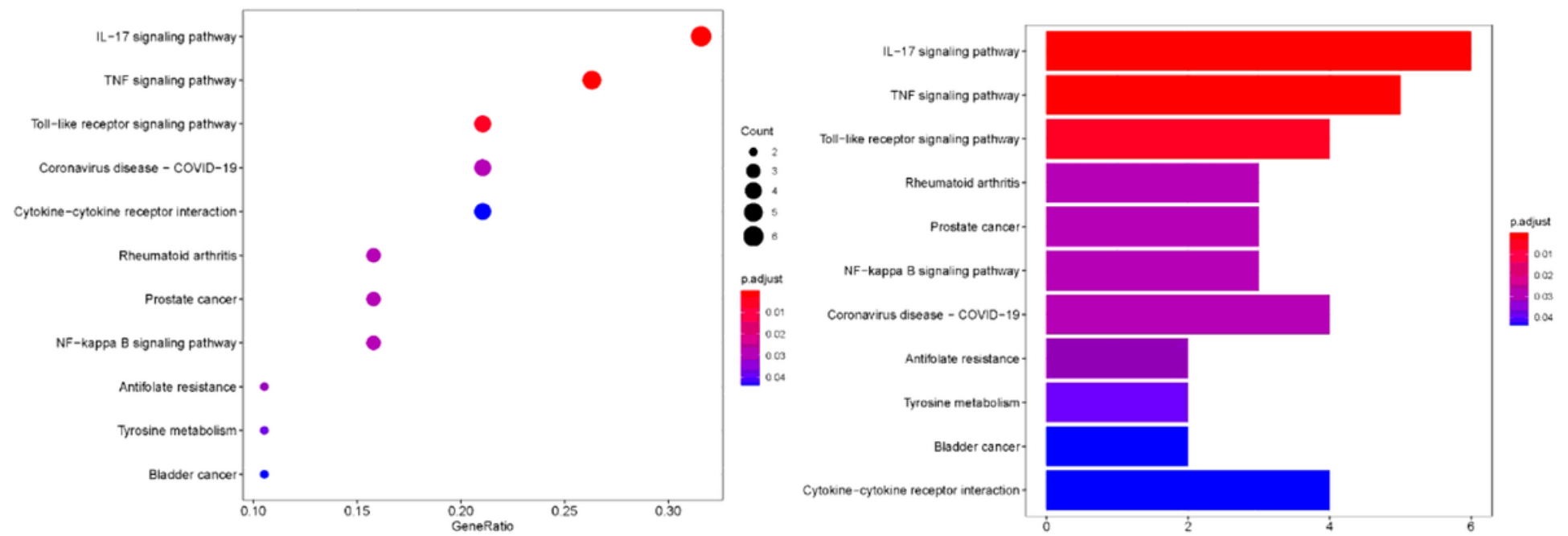
Figure 9

KEGG pathway enrichment analysis. The redder the color of the term, the smaller its P-value.

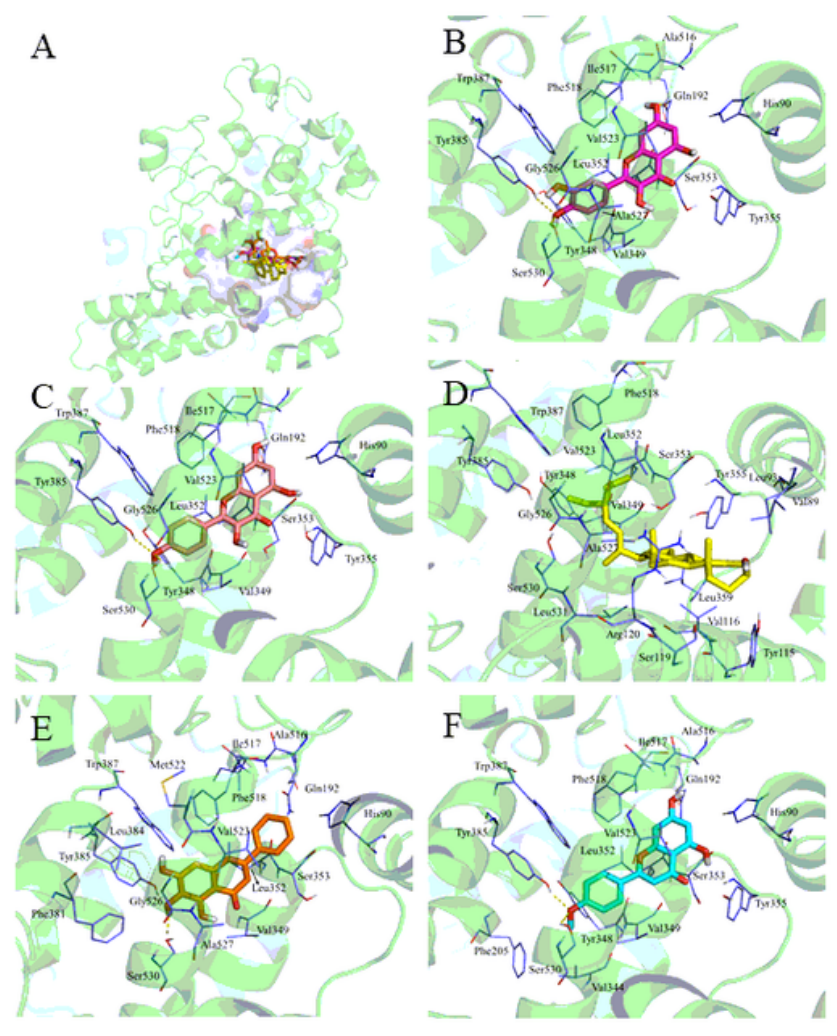

\section{Figure 10}

Docking models of XCHT active compounds (including quercetin, stigmasterol, kaempferol, baicalein, and acacetin) to potential target proteins. Note: Superposition of compounds binding to PTGS2 (A). The binding details of PTGS2 interacted with quercetin (B), kaempferol (C), Stigmasterol (D), baicalein (E), and acacetin $(F)$. The protein structure was presented as a green ribbon, and the interacted residues were shown as a blue line. Element colored the compounds, and the hydrogen bonds were displayed as a yellow dash.

\section{Supplementary Files}

This is a list of supplementary files associated with this preprint. Click to download.

- S1.xlsx

- S2.xlsx

- S3.xlsx

- S4.xlsx

- S5.xlsx 